\title{
Very Large Thermophase in Ferromagnetic Josephson Junctions
}

\author{
F. Giazotto, ${ }^{1, *}$ T. T. Heikkilä, ${ }^{2,3, \dagger}$ and F. S. Bergeret ${ }^{4,5,}$ \\ ${ }^{1}$ NEST, Instituto Nanoscienze-CNR and Scuola Normale Superiore, I-56127 Pisa, Italy \\ ${ }^{2}$ Department of Physics and Nanoscience Center, University of Jyväskylä, \\ P.O. Box 35 (YFL), FI-40014 University of Jyväskylä, Finland \\ ${ }^{3}$ Low Temperature Laboratory, Aalto University, P.O. Box 15100, FI-00076 AALTO, Finland \\ ${ }^{4}$ Centro de Física de Materiales (CFM-MPC), Centro Mixto CSIC-UPV/EHU, Manuel de Lardizabal 4, E-20018 San Sebastián, Spain \\ ${ }^{5}$ Donostia International Physics Center (DIPC), Manuel de Lardizabal 5, E-20018 San Sebastián, Spain
}

(Received 11 June 2014; revised manuscript received 8 September 2014; published 9 February 2015)

\begin{abstract}
The concept of thermophase refers to the appearance of a phase gradient inside a superconductor originating from the presence of an applied temperature bias across it. The resulting supercurrent flow may, in suitable conditions, fully counterbalance the temperature-bias-induced quasiparticle current therefore preventing the formation of any voltage drop, i.e., a thermovoltage, across the superconductor. Yet, the appearance of a thermophase is expected to occur in Josephson-coupled superconductors as well. Here, we theoretically investigate the thermoelectric response of a thermally biased Josephson junction based on a ferromagnetic insulator. In particular, we predict the occurrence of a very large thermophase that can reach $\pi / 2$ across the contact for suitable temperatures and structure parameters; i.e., the quasiparticle thermal current can reach the critical current. Such a thermophase can be several orders of magnitude larger than that predicted to occur in conventional Josephson tunnel junctions. In order to assess experimentally the predicted very large thermophase, we propose a realistic setup realizable with state-of-the-art nanofabrication techniques and well-established materials, based on a superconducting quantum interference device. This effect could be of strong relevance in several low-temperature applications, for example, for revealing tiny temperature differences generated by coupling the electromagnetic radiation to one of the superconductors forming the junction.
\end{abstract}

DOI: 10.1103/PhysRevLett.114.067001

PACS numbers: 74.50.+r, 74.25.F-, 85.25.Dq

Thermoelectric currents in superconductors are often shorted by supercurrents that generate a phase gradient, a thermophase, inside the superconductor. As suggested a long time ago by Ginzburg [1], a bimetallic superconducting loop constrains the possible phase gradients and allows the observation of thermoelectric effects via magnetic fields arising from circulating currents manipulated by temperature differences. Measurements of such circulating currents [2] were larger than that predicted by theory by several orders of magnitude, a discrepancy that is yet to be explained $[3,4]$.

In this Letter, we propose an alternative way to produce a very large thermophase in a superconducting quantum interference device (SQUID) loop consisting of a conventional superconductor in a spin-polarized contact with a superconductor-ferromagnet bilayer. The resulting thermophase can reach $\pi / 2$ across the contact, i.e., the quasiparticle thermal current can reach the critical current, and can be several orders of magnitude larger than that in conventional Josephson junctions $[5,6]$. Such a very large thermophase could be used for detecting tiny temperature differences, for instance, generated by radiation coupling to one of the superconductors.

We consider a Josephson junction [see Fig. 1(a)] consisting of two superconductors $S_{L}$ and $S_{R}$ tunnel coupled through a ferromagnetic insulator $(F I)$ and a nonmagnetic (I) barrier. The FI has different transmissivities for spin-up and spin-down electrons and therefore acts as a spin filter [7]. The interaction between the conduction electrons in $S_{L}$ with the localized magnetic moments of the $F I$ leads to an effective spin-splitting field $h$ in the left electrode that decays away from the interface over the superconducting coherence length $\xi_{0}$ [8]. The thin $I$ layer placed on the right side of the $F I$ prevents such a spin-splitting field from being induced in $S_{R}$ [9-11]. We assume that the thickness $t_{L}$ of $S_{L}$ is smaller than $\xi_{0}$ so that the induced $h$ is spatially uniform across the entire $S_{L}$ layer $[12,13]$. The junction is temperature biased so that $T_{L, R}$ is the temperature in $S_{L, R}$, respectively, and $\varphi^{\text {th }}$ denotes the phase difference between the superconducting order parameters induced by such a temperature difference. We focus on the static (i.e., time-independent) regime so that a dc Josephson current can flow in response to the applied thermal gradient but no thermovoltage develops across the junction.

In order to analyze the setup, we generalize the calculation of Ref. [17] to the case of two superconductors. The total electric current $I$ flowing through the junction is given by the sum of the quasiparticle $\left(I_{\mathrm{qp}}\right)$ and the Josephson contribution $\left(I_{J}\right)$ 
(a)
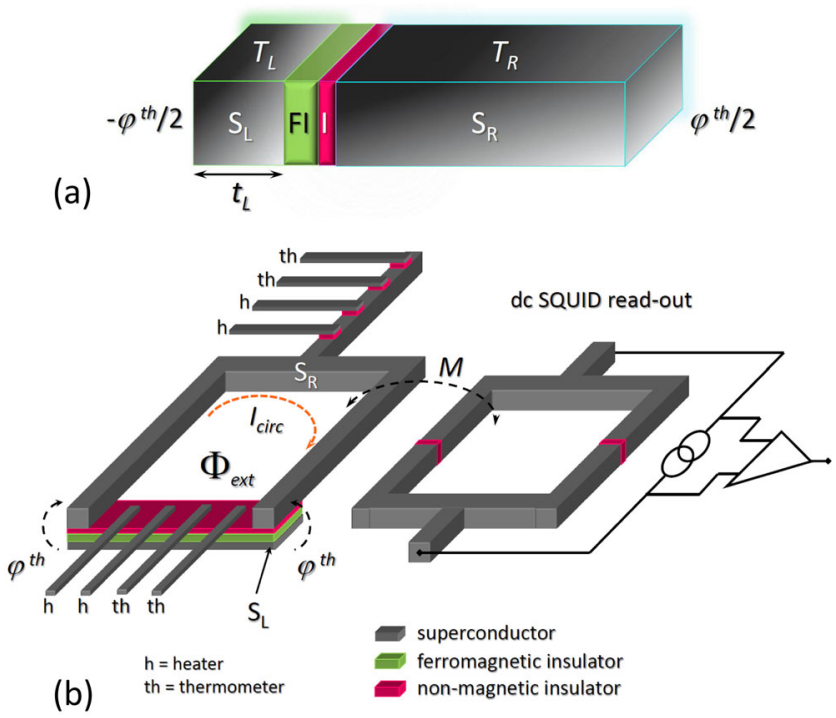

FIG. 1 (color online). Thermally biased ferromagnetic Josephson junction and the proposed experimental setup. (a) Sketch of a generic $S$-FI-I-S Josephson junction discussed in the text. It consists of two identical superconductors, $S_{L}$ and $S_{R}$, tunnel coupled by a ferromagnetic insulator $F I$ and a nonmagnetic barrier $I$. The direct contact between $F I$ and $S_{L}$ leads to an induced exchange field in the latter, while the nonmagnetic barrier prevents such a field from appearing in $S_{R} . T_{L}$ and $T_{R}$ are the temperature in $S_{L}$ and $S_{R}$, respectively, whereas $\varphi^{\text {th }}$ denotes the thermophase originated from thermally biasing the Josephson junction. $t_{L}$ is the thickness of $S_{L}$. (b) Scheme of a detection setup consisting of a temperature-biased superconducting quantum interference device based on the previous junction. Superconducting electrodes tunnel coupled to $S_{L}$ and $S_{R}$ serve either as heaters (h) or thermometers (th) and allow one to impose and detect a temperature gradient across the SQUID. The magnitude of the induced $\varphi^{\text {th }}$ can be determined by measuring variations of the supercurrent $\left(I_{\text {circ }}\right)$ circulating in the interferometer through a conventional dc SQUID inductively coupled to the first ring. $M$ denotes the mutual inductance between the loops, and $\Phi_{\text {ext }}$ is the external applied magnetic flux.

$$
I=I_{\mathrm{qp}}+I_{J}=I_{\mathrm{qp}}+I_{c} \sin \varphi^{\text {th }},
$$

where $I_{c}$ is the critical supercurrent. The current contribution proportional to $\cos \varphi^{\text {th }}$ [18] does not contribute, since it does not possess any thermoelectric response, and it would require a finite voltage. The explicit forms for $I_{\mathrm{qp}}$ and $I_{c}$ in Eq. (1) can be obtained from the expressions for the current through a spin-filter barrier with polarization $P$ [19-21],

$$
I_{\mathrm{qp}}=\frac{P}{2 e R_{T}} \int_{-\infty}^{\infty} d \varepsilon N_{L}^{-}(\varepsilon) N_{R}(\varepsilon)\left[f_{L}(\varepsilon)-f_{R}(\varepsilon)\right]
$$

and

$$
\begin{aligned}
I_{c}= & -\frac{\sqrt{1-P^{2}}}{2 e R_{T}} \int_{-\infty}^{\infty} d \varepsilon\left[\operatorname{Re} M_{L}(\varepsilon) \operatorname{Im} F_{R}(\varepsilon) f_{L}(\varepsilon)\right. \\
& \left.+\operatorname{Im} M_{L}(\varepsilon) \operatorname{Re} F_{R}(\varepsilon) f_{R}(\varepsilon)\right] .
\end{aligned}
$$

In Eqs. (2) and (3), $N_{L}^{-}(\varepsilon)=\left[N_{L}(\varepsilon+h)-N_{L}(\varepsilon-h)\right] / 2$, $N_{L, R}(\varepsilon)=\left|\operatorname{Re}\left[(\varepsilon+i \Gamma) / \sqrt{(\varepsilon+i \Gamma)^{2}-\Delta_{L, R}^{2}}\right]\right|$ is the normalized BCS density of states in $S_{L, R}, M_{L}(\varepsilon)=\left[F_{L}(\varepsilon+h)+\right.$ $\left.F_{L}(\varepsilon-h)\right] / 2, F_{L, R}(\varepsilon)=\Delta_{L, R} / \sqrt{(\varepsilon+i \Gamma)^{2}-\Delta_{L, R}^{2}}, f_{L, R}(\varepsilon)=$ $\tanh \left(\varepsilon / 2 k_{B} T_{L, R}\right)$, and $\Delta_{L, R}$ is the energy gap in $S_{L, R}$ that has to be determined self-consistently due to the presence of $h$ and finite $T_{L / R}$. For ideal superconductors $\Gamma \rightarrow 0^{+}$[22]; we have checked that the value $\Gamma=10^{-4} \Delta_{0}$ chosen in our numerics negligibly affects the results. Above, $\Delta_{0}$ denotes the zero-temperature, zero-exchange field superconducting energy gap. Furthermore, $e$ is the electron charge, $k_{B}$ is the Boltzmann constant, and $R_{T}$ is the normal-state resistance of the junction. Equation (2) shows that for a nonvanishing $I_{\mathrm{qp}}$ to exist (i) a finite $h$ should be induced in one of the superconductors, and (ii) $P$ has to be finite [17].

In an "electrically open" configuration, the total charge current has to vanish, $I=0$. Therefore, in order to ensure a vanishing thermovoltage across the junction, the quasiparticle current $I_{\mathrm{qp}}$ induced by the temperature gradient has to be canceled by an opposite dc supercurrent $I_{J}$. This cancellation is the origin of the thermophase, which is defined as

$$
\varphi^{\mathrm{th}}=\arcsin \left(-\frac{I_{\mathrm{qp}}}{I_{c}}\right) .
$$

This thermophase is thus a measure of the amplitude of the thermoelectric effect at the contact between the superconductors. In the "electrically closed" configuration, $\varphi^{\text {th }}$ is necessarily no longer the phase difference across the junction. In this case, Eq. (4) should be viewed as a definition, characterizing the relative magnitude of the quasiparticle current vs the critical current of the junction. Below, we discuss a scheme for measuring this thermophase.

Let us analyze the behavior of $I_{\mathrm{qp}}$ and of $I_{c}$ under thermal bias. Figures 2(a) and 2(b) show $I_{\mathrm{qp}}$ vs $T_{L}$ and $T_{R}$, respectively, when the temperature of the other electrode is fixed to $0.1 T_{c}$. Here, $T_{c}$ is the superconducting critical temperature in the absence of $h$, which we assume, for simplicity, to be the same for both superconductors. By varying $T_{L}$ and depending on the temperature range, the thermocurrent can be either "positive," i.e., flowing according to the thermal gradient set across the junction, or "negative." The sign of the current can be ascribed to a more electron- or holelike contribution to thermoelectric transport, respectively. The amplitude of $I_{\mathrm{qp}}$ is, in general, larger for larger $h$ and drops eventually to zero at the temperature for which $\Delta_{L}$ vanishes. The value of such a critical temperature depends on the value of $h$. By contrast, Fig. 2(b) shows that the thermocurrent does not change sign if $T_{L}$ is held constant and $T_{R}\left(>T_{L}\right)$ is varied. The amplitude of $I_{\mathrm{qp}}$ in this case is larger than that obtained 

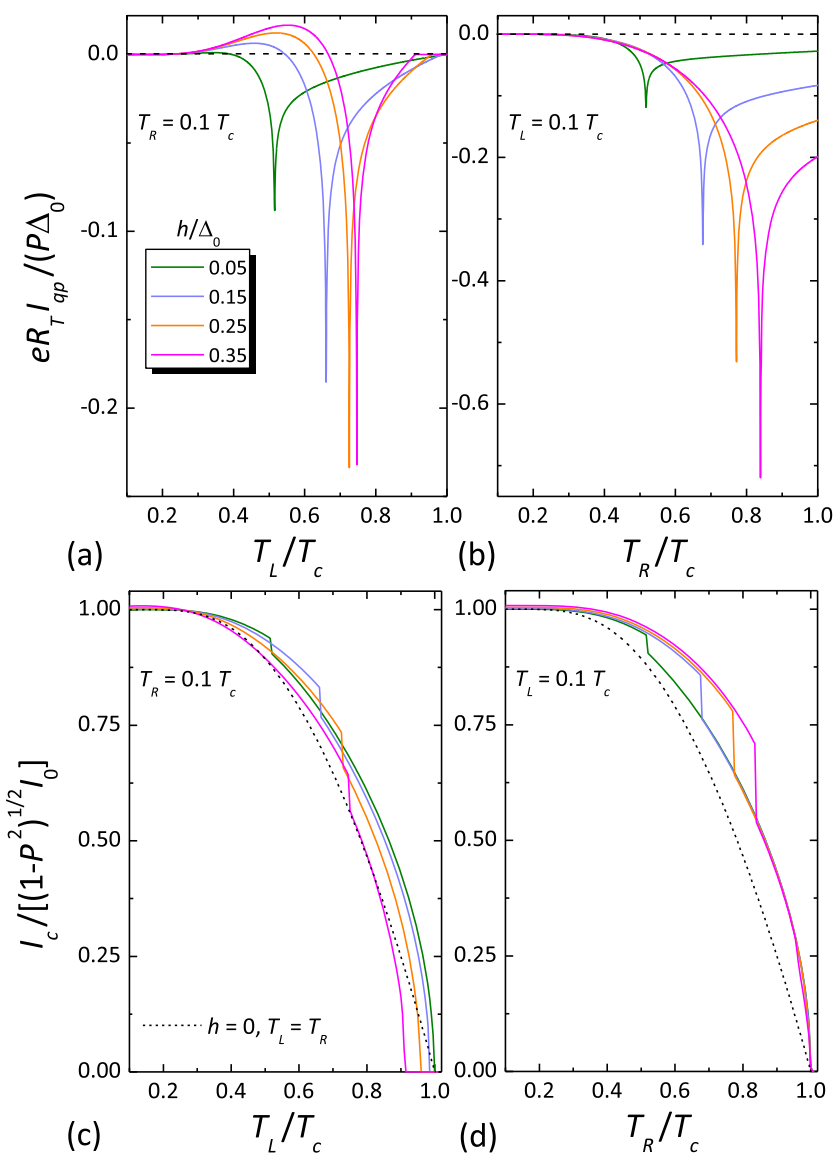

FIG. 2 (color online). Quasiparticle and Josephson critical currents under thermal-bias conditions for different values of the exchange field. Quasiparticle current $I_{\mathrm{qp}}$ (a) vs $T_{L}$ at $T_{R}=$ $0.1 T_{c}$ and (b) vs $T_{R}$ at $T_{L}=0.1 T_{c}$; Josephson critical current $I_{c}$ (c) vs $T_{L}$ at $T_{R}=0.1 T_{c}$ and (d) vs $T_{R}$ at $T_{L}=0.1 T_{c}$. Dashdotted curves in panels (c) and (d) are calculated for $h=0$ and $T_{L}=T_{R} . \Delta_{0}$ denotes the zero-exchange field, zero-temperature superconducting energy gap corresponding to the critical temperature $T_{c} \approx \Delta_{0} / 1.764 k_{B}$ whereas $I_{0}$ is the zero-exchange field, zero-temperature Josephson critical current.

by varying $T_{L}>T_{R}$ and is finite at $T_{c}$. Notably, the quasiparticle characteristics exhibit sharp dips positioned at the temperatures satisfying the condition

$$
\left|\Delta_{L}\left(T_{L}, h\right)-\Delta_{R}\left(T_{R}\right)\right|=h .
$$

The large values obtained by $I_{\mathrm{qp}}$ are the origin of a very large thermophase achievable in ferromagnetic Josephson junctions.

Similarly, the lower panels of Fig. 2 display the behavior of $I_{c}$ vs $T_{R}$ and $T_{L}$ by holding the other electrode at $0.1 T_{c}$. The $I_{c}(T)$ curves differ drastically from those obtained for $h=0$ at $T_{L}=T_{R}$ (dash-dotted curves). In particular, at a low enough temperature $I_{c}$ gets larger by increasing $h$. This remarkable effect corresponds to the supercurrent enhancement discussed in Ref. [23], which occurs even for $P=0$. We stress that the $I_{c}$ strengthening joined with the sharp
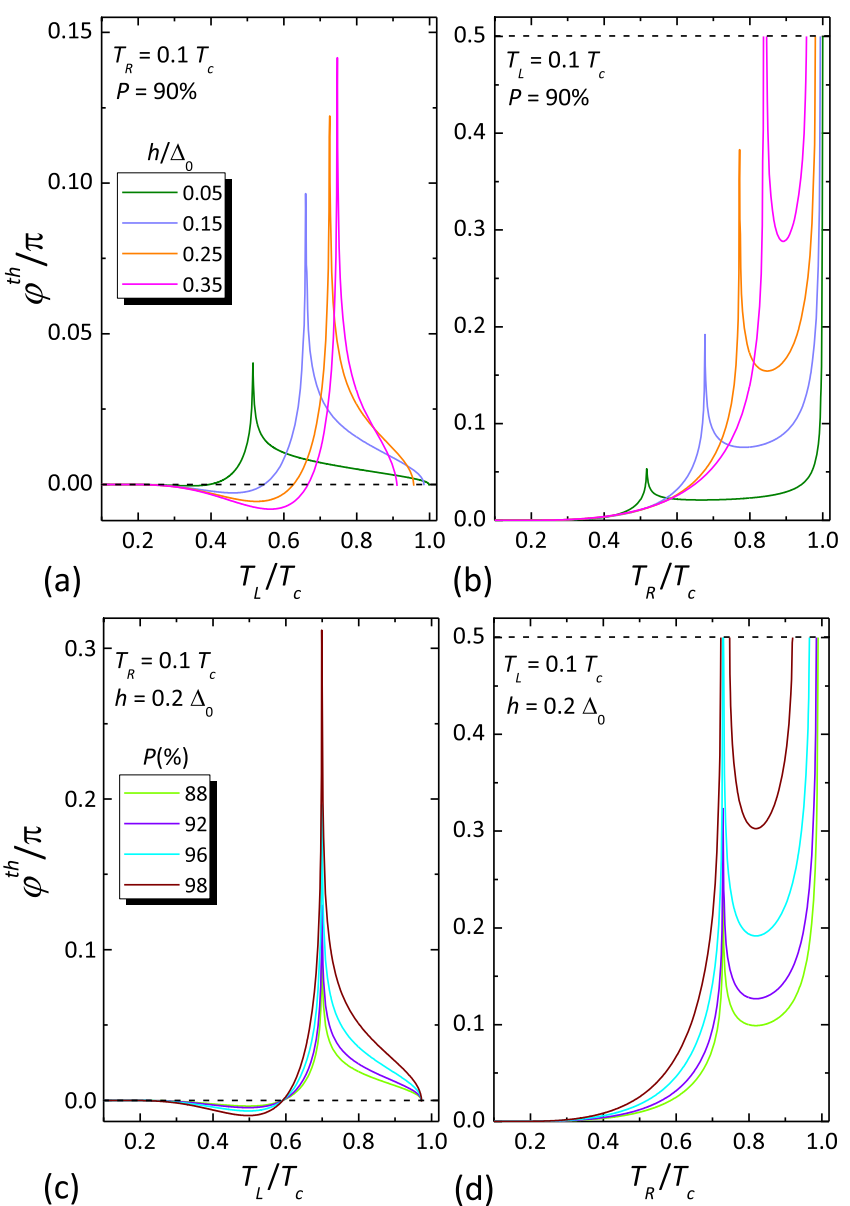

FIG. 3 (color online). Behavior of the thermophase in a ferromagnetic Josephson junction. (a) Thermophase $\varphi^{\text {th }}$ vs $T_{L}$ calculated for several values of the exchange field $h$ at $T_{R}=$ $0.1 T_{c}$ and $P=90 \%$. (b) $\varphi^{\text {th }}$ vs $T_{R}$ calculated at $T_{L}=0.1 T_{c}$ and $P=90 \%$ for the same exchange field values as in panel (a). (c) $\varphi^{\text {th }}$ vs $T_{L}$ calculated for several values of the polarization $P$ of the spin-filter barrier at $T_{R}=0.1 T_{c}$ and $h=0.2 \Delta_{0}$. (d) $\varphi^{\text {th }}$ vs $T_{R}$ calculated at $T_{L}=0.1 T_{c}$ and $h=0.2 \Delta_{0}$ for the same $P$ values as in panel (c). $\varphi^{\text {th }}$ is not defined in the temperature regions where $\left|I_{\mathrm{qp}}\right|$ exceeds $I_{c}$.

jumps appearing at those temperatures where Eq. (5) holds are a manifestation of an exchange field induced in $S_{L}$ and of a nonequilibrium condition stemming from the thermal bias [23].

The thermophase $\varphi^{\text {th }}$ is obtained from Eq. (4). Figures 3(a) and 3(b) show the dependence of $\varphi^{\text {th }}$ on $T_{L}$ and $T_{R}$, respectively, when the other electrode is kept at $0.1 T_{c}$. We have chosen a reasonable polarization of the barrier $(P=90 \%)$ and moderate $h$ values easily achievable with present-day experiments $[7,10]$. We find that $\varphi^{\text {th }}$ can be very large, close to $\pi / 2$ for $h \gtrsim 0.3 \Delta_{0}$. This substantial effect has to be compared to the minute one achievable in conventional nonferromagnetic Josephson tunnel junctions where $\varphi^{\text {th }} \sim 10^{-4}$ is expected $[5,6]$. We also notice the presence of temperature regions where $\varphi^{\text {th }}$ is not defined, since $I_{\mathrm{qp}}$ may 
exceed $I_{c}$ [see Fig. 3(b)]. In this case, a finite dc voltage is induced across the contact.

The impact of $P$ on $\varphi^{\text {th }}$ is shown in Figs. 3(c) and 3(d) where we set $h=0.2 \Delta_{0}$, and the temperature in the superconductors is varied similarly to Figs. 3(a) and 3(b), respectively. The increase of $P$ leads to a sizable thermophase enhancement, as $I_{\mathrm{qp}} / I_{c} \propto P / \sqrt{1-P^{2}} \quad$ [see Eqs. (2)-(4)]. From Fig. 3, it becomes clear that large values of $\varphi^{\text {th }}$ can be obtained more easily by increasing $T_{R}$ while keeping $S_{L}$ at a low temperature, consistently with the $I_{\mathrm{qp}}\left(T_{R}\right)$ dependence shown in Fig. 2(b). The full behavior of $\varphi^{\text {th }}$ vs $T_{R}$ for $T_{L}=0.1 T_{c}$ is displayed in the color plots of Fig. 4. Specifically, we set $P=96 \%$ and varied $h$ in panel (a), whereas in panel (b) we set $h=0.3 \Delta_{0}$ and varied $P$. The figures show that a sizeable $\varphi^{\text {th }}$ can be obtained in a rather large range of parameters and may provide a valuable tool for tailoring optimized junctions where $\varphi^{\text {th }}$ is maximized.

To assess experimentally the predicted very large thermophase, we propose the setup depicted in Fig. 1(b). It consists of a SQUID, including a FI and comprising a

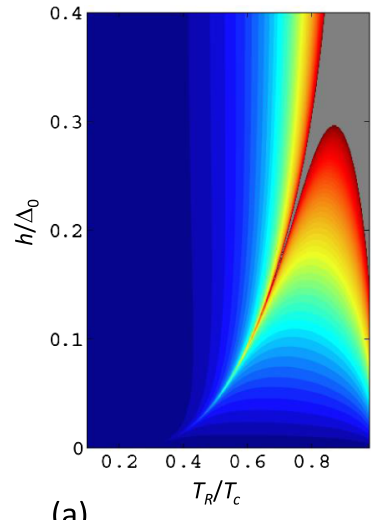

(a)

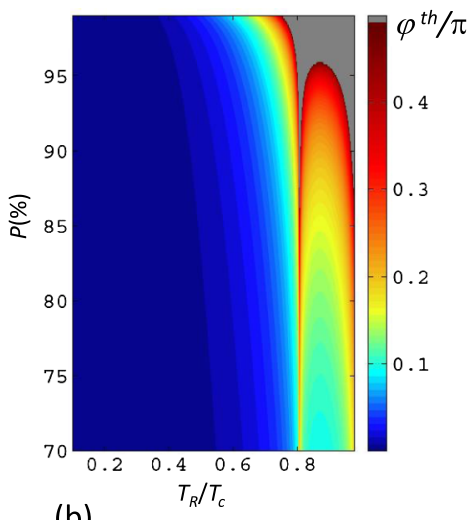

(b)

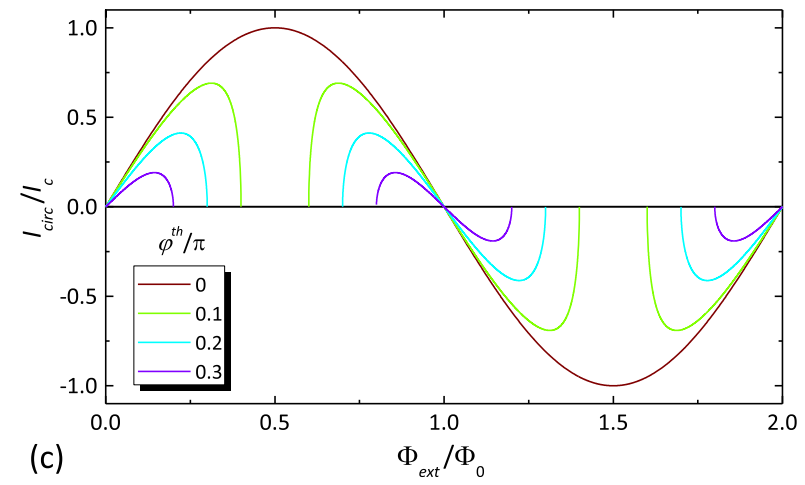

FIG. 4 (color online). Full behavior of the thermophase and response of the circulating current in a temperature-biased FI SQUID. (a) Color plot of the thermophase $\varphi^{\text {th }}$ vs $T_{R}$ and $h$ calculated at $T_{L}=0.1 T_{c}$ for $P=96 \%$. (b) Color plot of $\varphi^{\text {th }}$ vs $T_{R}$ and $P$ calculated at $T_{L}=0.1 T_{c}$ for $h=0.3 \Delta_{0}$. (c) Circulating current $I_{\text {circ }}$ vs external magnetic flux $\Phi_{\text {ext }}$ for a few values of the thermophase in a symmetric SQUID. number of superconducting tunnel junctions that can either heat or perform accurate electron thermometry [24]. From the materials side, $F I$ s such as $\mathrm{EuO}$ or EuS (providing $P$ up to $98 \%$ ) [25] in contact with superconducting $\mathrm{Al}$ appear as ideal candidates for the implementation of the structure that can be realized with standard lithographic techniques. The ratio $h / \Delta_{0}$ in such structures depends on the thickness of the Al layer and quality of the contact. In the superconducting state of $\mathrm{Al}$, values ranging from $h / \Delta_{0} \approx 0.2$ up to 0.6 have been reported $[10,11,14,15]$. Alternatively, GdN barriers in combination with $\mathrm{Nb}$ or $\mathrm{NbN}$ could be used as well [26,27].

In the SQUID, the thermophase $\varphi_{1 / 2}^{\text {th }}$ developed across the two junctions results into a nonzero total circulating current $I_{\text {circ }}$. In the absence of an external flux and for negligible loop inductance, the amplitude of the total circulating current is given by

$$
I_{\text {circ }}=\frac{I_{c 1} I_{c 2}}{I_{c 1}+I_{c 2}}\left|\sin \left(\varphi_{1}^{\mathrm{th}}\right)-\sin \left(\varphi_{2}^{\mathrm{th}}\right)\right|,
$$

where $I_{c i}$ is the critical current for contact $i=1,2$. Since $\varphi_{i}^{\text {th }}$ depends on the ratio of the quasiparticle and critical supercurrents, an asymmetry of the resistances between the contacts would not cause a circulating current. However, replacing one of the junctions, for example, 2, by a conventional SIS junction would set $\varphi_{2}^{\text {th }} \approx 0$ and therefore would lead to a large $I_{\text {circ }}$ even without an external flux.

An alternative way to measure the thermophase is to consider the case of a finite external flux $\Phi_{\text {ext }}$. If the SQUID junctions are identical, $I_{\text {circ }}$ can be written as

$$
I_{\text {circ }}=I_{c}\left(\sin \varphi_{1}-\sin \varphi^{\text {th }}\right)=-I_{c}\left(\sin \varphi_{2}-\sin \varphi^{\text {th }}\right) .
$$

In Eq. (7), $I_{\text {circ }}$ is written as the sum of a magnetic-fluxdependent part and the one of thermoelectric origin, and the second equality expresses the conservation of the supercurrent circulating along the loop through each junction of the SQUID. In the limit of negligible ring inductance Eq. (7) can be analytically solved for $I_{\text {circ }}$ by imposing fluxoid quantization, $\varphi_{2}-\varphi_{1}=2 \pi \Phi_{\text {ext }} / \Phi_{0}$, where $\Phi_{0}=$ $2.067 \times 10^{-15} \mathrm{~Wb}$ is the flux quantum. The result for $I_{\text {circ }}$ is thus

$$
I_{\text {circ }}=I_{c} \sin \left(\frac{\pi \Phi_{\text {ext }}}{\Phi_{0}}\right) \operatorname{Re}\left[\sqrt{1-\frac{\sin ^{2} \varphi^{\text {th }}}{\cos ^{2}\left(\frac{\pi \Phi_{\text {ext }}}{\Phi_{0}}\right)}},,\right.
$$

which holds for $I_{\mathrm{qp}} \leq I_{c}$ [see Eq. (4)]. For $\varphi^{\text {th }}=0$, Eq. (8) reduces to

$$
I_{\text {circ }}=I_{c} \sin \left(\frac{\pi \Phi_{\mathrm{ext}}}{\Phi_{0}}\right) .
$$

As shown in Fig. 4(c), the presence of a finite thermophase gives rise to regions of flux close to $\Phi_{0} / 2$ and $3 \Phi_{0} / 2$ where 
$I_{\text {circ }}$ vanishes because the thermoelectric current becomes larger than the effective critical current of the SQUID. The presence of these regions is a direct evidence of the thermophase. The circulating current can be detected through a conventional de SQUID inductively coupled to the first loop [see Fig. 1(b)] so that $\Phi_{\text {SQUID }} \sim M I_{\text {circ }}$, where $\Phi_{\text {SQUID }}$ is the magnetic flux induced in the readout SQUID and $M$ is the mutual inductance coefficient. For instance, for typical values of $I_{c}=1 \mu \mathrm{A}$ and $M=10 \mathrm{pH}, \Phi_{\text {SQUID }}$ up to $\sim 10^{-17} \mathrm{~Wb} \approx 5 \times 10^{-3} \Phi_{0}$ can be generated with a proper temperature bias, as in Figs. 2-4. This can be well detected with standard SQUIDs, which routinely provide magnetic flux sensitivities $\sim 10^{-6} \Phi_{0} / \sqrt{\mathrm{Hz}}$ [28].

In conclusion, we have predicted the occurrence of a very large thermophase in thermally biased Josephson junctions based on FIs. This sizeable effect can be detected in a structure realizable with current state-of-the-art nanofabrication techniques and well-established materials. Besides shedding light onto fundamental problems related to the thermoelectric response of superconductors and exotic weak links in the Josephson regime, the very sharp thermophase response (see Fig. 3) combined with the low heat capacity of superconductors could allow the realization of ultrasensitive radiation detectors [24] where radiation-induced heating of one of the superconductors is detected via the thermophase. The presence of magnetic material also allows for adding a new control parameter to the experimental investigation of the coherent manipulation of heat flow at the nanoscale [29-32].

F. G. acknowledges the Marie Curie Initial Training Action (ITN) Q-NET 264034 and the European Research Council under the European Union's Seventh Framework Programme (FP7/2007-2013)/ERC Grant agreement No. 615187-COMANCHE for partial financial support. The work of F.S.B has been supported by the Spanish Ministry of Economy and Competitiveness under Project No. FIS2011-28851-C02-02, and the work of T. T. H. has been supported by the ERC (Grant No. 240362-Heattronics) and the Academy of Finland through its Center of Excellence program.

*giazotto@sns.it

Tero.T.Heikkila@jyu.fi

*sebastian_bergeret@ehu.es

[1] V. L. Ginzburg, Zh. Eksp. Teor. Fiz. 14, 177 (1944) [Sov. Phys. JETP 8, 148 (1944)].

[2] D. J. Van Harlingen, D. F. Heidel, and J. C. Garland, Phys. Rev. B 21, 1842 (1980).

[3] V. L. Ginzburg, Rev. Mod. Phys. 76, 981 (2004).

[4] Y. M. Galperin, V. L. Gurevich, V.I. Kozub, and A. L. Shelankov, Phys. Rev. B 65, 064531 (2002).

[5] G. D. Guttman, B. Nathanson, E. Ben-Jacob, and D. J. Bergman, Phys. Rev. B 55, 12691 (1997).
[6] A. D. Smith, M. Tinkham, and W. J. Skocpol, Phys. Rev. B 22, 4346 (1980).

[7] J. S. Moodera, T. S. Santos, and T. Nagahama, J. Phys. Condens. Matter 19, 165202 (2007).

[8] T. Tokuyasu, J. A. Sauls, and D. Rainer, Phys. Rev. B 38, 8823 (1988).

[9] X. Hao, J. S. Moodera, and R. Meservey, Phys. Rev. B 42 , 8235 (1990).

[10] B. Li, G.-X. Miao, and J. S. Moodera, Phys. Rev. B 88, 161105(R) (2013).

[11] B. Li, N. Roschewsky, B. A. Assaf, M. Eich, M. EpsteinMartin, D. Heiman, M. Münzenberg, and J. S. Moodera, Phys. Rev. Lett. 110, 097001 (2013).

[12] F. Giazotto and F. Taddei, Phys. Rev. B 77, 132501 (2008).

[13] We note that ultrathin superconducting $\mathrm{Al}$ films (i.e., with thickness $\left.t_{L} \sim 2-3 \mathrm{~nm}\right)$ can be routinely grown $[10,11,14,15]$, and typically provide a critical temperature $\sim 3 \mathrm{~K}$ with a coherence length $\xi_{0} \sim 15 \mathrm{~nm}$ [15]. For such films, therefore, the condition $t_{L}<\xi_{0}$, which insures spatial uniformity of the induced exchange field $h$ across the whole $S_{L}$ layer, can be easily met.

[14] Y. M. Xiong, S. Stadler, P. W. Adams, and G. Catelani, Phys. Rev. Lett. 106, 247001 (2011).

[15] T. J. Liu, J. C. Prestigiacomo, and P. W. Adams, Phys. Rev. Lett. 111, 027207 (2013).

[16] F. S. Bergeret, A. F. Volkov, and K. B. Efetov, Phys. Rev. Lett. 86, 3140 (2001).

[17] A. Ozaeta, P. Virtanen, F. S. Bergeret, and T. T. Heikkilä, Phys. Rev. Lett. 112, 057001 (2014).

[18] A. Barone and G. Paterno, Physics and Applications of the Josephson Effect (Wiley-Interscience, New York, 1982).

[19] F. S. Bergeret, A. Verso, and A. F. Volkov, Phys. Rev. B 86, 060506(R) (2012).

[20] F. S. Bergeret, A. Verso, and A. F. Volkov, Phys. Rev. B 86, 214516 (2012).

[21] P. Machon, M. Eschrig, and W. Belzig, Phys. Rev. Lett. 110, 047002 (2013).

[22] R. C. Dynes, J. P. Garno, G. B. Hertel, and T. P. Orlando, Phys. Rev. Lett. 53, 2437 (1984).

[23] F. S. Bergeret and F. Giazotto, Phys. Rev. B 89, 054505 (2014).

[24] F. Giazotto, T. T. Heikkilä, A. Luukanen, A. M. Savin, and J. P. Pekola, Rev. Mod. Phys. 78, 217 (2006).

[25] T. S. Santos, J. S. Moodera, K. V. Raman, E. Negusse, J. Holroyd, J. Dvorak, M. Liberati, Y. U. Idzerda, and E. Arenholz, Phys. Rev. Lett. 101, 147201 (2008).

[26] K. Senapati, M. G. Blamire, and Z. H. Barber, Nat. Mater. 10, 849 (2011).

[27] A. Pal, Z.H. Barber, J. W. A. Robinson, and M. G. Blamire, Nat. Commun. 5, 3340 (2014).

[28] The SQUID Handbook, edited by J. Clarke and A. I. Braginski (Wiley-VCH, Weinheim, Germany, 2004).

[29] F. Giazotto and M. J. Martínez-Pérez, Nature (London) 492, 401 (2012).

[30] M. J. Martínez-Pérez, P. Solinas, and F. Giazotto, J. Low Temp. Phys. 175, 813 (2014).

[31] M. J. Martínez-Pérez and F. Giazotto, Nat. Commun. 5, 3579 (2014).

[32] M. Meschke, W. Guichard, and J. P. Pekola, Nature (London) 444, 187 (2006). 